Valentin A. Skvortsov, Department of Mathematics, Moscow State

University, Moscow 119899, Russia

\title{
CONTINUITY OF $\delta$-VARIATION AND CONSTRUCTION OF CONTINUOUS MAJOR AND MINOR FUNCTIONS FOR THE PERRON INTEGRAL
}

\begin{abstract}
It is proved that if a function is continuous on an interval, then its variations on a set taken relative to some covering relations are also continuous as interval-functions. This result is applied to a direct construction of continuous Perron major and minor functions of Henstock integrable function.
\end{abstract}

The Henstock Integral with respect to a derivation base is known to be equivalent to the Perron Integral defined with respect to the same base. (See [9] for an abstract setting.) But the Perron Integral is understood in this theory to be defined using major and minor functions which are not necessarily continuous with respect to the base. The question of whether we get an equivalent definition by restricting the major and minor functions to be continuous is still an open one in a general case. (See [1, p. 202] and [2].) This question is closely related to the continuity property of a variation which arises in the theory of Henstock integration.

The classical Perron Integral on an interval in the real line $\mathbb{R}$ is defined by means of continuous major and minor functions. The well-known proof (see [11]) of the fact that this definition is equivalent to the one without the continuity condition is based on the descriptive theory of the Denjoy Integral and we are not aware of any satisfactory proof of this equivalence having been presented which uses just elementary properties of the Henstock integral. (We shall indicate below a gap in the proof of related theorems in [4] and [8]). A

\footnotetext{
Key Words: Perron integral, Henstock integral, covering relation, variation

Mathematical Reviews subject classification: Primary: 26A39; Secondary: 26A42, $26 \mathrm{~A} 45$

Received by the editors March 14, 1995
} 
recent proof given in [9] is also based in fact on showing first that the primitive is an ACG function, i.e. on Denjoy's approach. But some ideas of that proof can be adapted to a direct construction. In particular we are borrowing here from [9] the idea of using the variations defined by left and right covering relations.

In this note we start with proving that if a function is continuous on an interval in $\mathbb{R}$, then its $\delta$-variation on a set taken relative to some covering relation is also continuous as an interval-function. Then we apply this result to construct directly continuous major and minor functions of a Henstock integrable function. By a direct construction we mean here the same kind of construction, based on variation, that is usually used in a direct proof of the fact that a Henstock integrable function is Perron integrable (see [6], [10]).

We need some facts and definitions (see [10] and [13]).

A covering relation is a subset $B$ of the product $\mathcal{I} \times R$ where $\mathcal{I}$ is the collection of all nondegenerate closed intervals. If $E$ is a set in $\mathbb{R}$ we set

$$
\begin{aligned}
B(E) & =\{(I, x) \in B: I \subset E\}, \\
B[E] & =\{(I, x) \in B: x \in E\} .
\end{aligned}
$$

We shall always suppose here that $x \in I$ for any $(I, x) \in B$. If a function $\delta(x)>0$ is defined on a set $E$, we let

$$
B_{\delta}=\{(I, x) \in B: I \subset(x-\delta(x), x+\delta(x))\} .
$$

In particular this defines $B_{\delta}[E]$ and $B_{\delta}(E)$. If $x$ is fixed, $B[\{x\}]$ is sometimes called a derivation base at $x$. We shall suppose that for any $x$ and for any $\delta>0$ we have $B_{\delta}[\{x\}] \neq \emptyset$ (Vitali property).

If at any point $x \in E$ for some $\delta(x)>0$

$$
B_{\delta}[E]=\{(I, x): I \subset(x-\delta(x), x+\delta(x)), x \in E\},
$$

we say that $B_{\delta}$ is a full covering relation on $E$. A finite subset $\pi$ of $B_{\delta}[E]$ is called a partition of $B_{\delta}$ on $E$ if the intervals $I_{1}$ and $I_{2}$ for distinct $\left(I_{1}, x_{1}\right)$ and $\left(I_{2}, x_{2}\right)$ belonging to $\pi$ do not overlap.

All functions we consider are real-valued. If $F$ is a point-function, we shall use the same notation for its associated interval-function, i.e. if $I=[c, d]$, then we put $F(I)=F(d)-F(c)$.

Let a function $F$ be defined on an interval $A$ and let a relation $B_{\delta}$ and a set $E \subset R$ be fixed. We define

$$
V_{\delta}(A)=V\left(B_{\delta}, F, E, A\right)=\sup \left\{\sum_{(I, x) \in \pi}|F(I)|: \pi \subset B_{\delta}[E] \cap B_{\delta}(A)\right\}
$$


and call this the $\delta$-variation over $B$ of the function $F$ on $E \cap A$. If $B_{\delta}[E] \cap$ $B_{\delta}(A)=\emptyset$, we agree that the variation vanishes. If $E$ and $\delta$ are fixed, $V_{\delta}(A)$ is obviously a nonnegative superadditive interval-function of $A$.

Proposition 1 Let $F$ be a continuous point-function on an interval $[a, b]$, let a set $E \subset[a, b]$, a covering relation $B$ and a function $\delta(x)>0$ on $E$ be fixed and let $V_{\delta}([a, b])$ be finite. Then the $\delta$-variation $V_{\delta}(A)$ being considered as an interval-function of $A \subset[a, b]$ is continuous at each point $x \in[a, b]$, i.e. for any $x \in[a, b]$ and any $\varepsilon>0$ there exists $\eta>0$ so that $V_{\delta}(A)<\varepsilon$ if $x \in A \subset[x-\eta, x+\eta] \cap[a, b]$.

Proof. An analogous proposition for a special symmetric case was proved in [12] and we employ a similar argument here. Take any $x \in[a, b]$ and any $\varepsilon>0$. Since $F$ is continuous, we can choose $\eta_{1}>0$ such that

$$
|F(I)|<\frac{\varepsilon}{4} \text { for each interval } I \subset[a, b] \text { with }|I|<\eta_{1} .
$$

Take $J=\left[x-\frac{1}{2} \eta_{1}, x+\frac{1}{2} \eta_{1}\right] \cap[a, b]$. There exists a partition $\pi=\left\{\left(I_{i}, x_{i}\right)\right\} \subset$ $B_{\delta}[E \cap J] \cap B_{\delta}(J)$ so that

$$
V_{\delta}(J) \geq \sum_{\left(I_{i}, x_{i}\right) \in \pi}\left|F\left(I_{i}\right)\right|>V_{\delta}(J)-\frac{\varepsilon}{4}
$$

Now we can choose a desirable $\eta>0$. Take it so that $\eta<\frac{1}{2} \eta_{1}$ and that $(x-\eta, x+\eta)$ meets only those intervals $I_{i}$ which contain $x$. There are no more than two such intervals. Let them be $I^{\prime}$ and $I^{\prime \prime}$. Note that $\left|F\left(I^{\prime}\right)\right|$ and $\left|F\left(I^{\prime \prime}\right)\right|$ are both less than $\frac{\varepsilon}{4}$, (see [1]).

Let $\pi_{1}=\pi \backslash\left\{\left(I^{\prime}, x^{\prime}\right),\left(I^{\prime \prime}, x^{\prime \prime}\right)\right\}$. Then

$$
\sum_{(I, x) \in \pi_{1}}|F(I)|>\sum_{(I, x) \in \pi}|F(I)|-\frac{\varepsilon}{2} .
$$

Let $A$ denote any interval with $x \in A$ and $A \subset J_{1}=[x-\eta, x+\eta] \cap[a, b]$. We show that $V_{\delta}(A)<\varepsilon$. If not, then $V_{\delta}\left(J_{1}\right) \geq V_{\delta}(A) \geq \varepsilon$. This yields a contradiction. Indeed, take a partition $\pi_{2} \subset B_{\delta}\left[E \cap J_{1}\right] \cap B_{\delta}\left(J_{1}\right)$ such that

$$
\sum_{(I, x) \in \pi_{2}}|F(I)|>V_{\delta}\left(J_{1}\right)-\frac{\varepsilon}{4} \geq \frac{3 \varepsilon}{4} .
$$

Then together with (2) and (3) we must have

$$
V_{\delta}(J) \geq \sum_{(I, x) \in \pi_{1}}|F(I)|+\sum_{(I, x) \in \pi_{2}}|F(I)|>V_{\delta}(J)-\frac{\varepsilon}{4}-\frac{\varepsilon}{2}+\frac{3 \varepsilon}{4}=V_{\delta}(J)
$$


which is impossible. Thus we have proved the continuity of $V_{\delta}(A)$ at each point of $[a, b]$.

Note that $V_{\delta}(A)$ being superadditive need not be additive. We can see this from the following example. Let $x_{0}$ be an isolated point of $E$, let also $[a, b]=[a, c] \cup[c, b], c<x_{0}<b,\left(x_{0}-\delta\left(x_{0}\right), x_{0}\right] \cap E=\left\{x_{0}\right\},\left(I_{0}, x_{0}\right) \in B_{\delta}\left[\left\{x_{0}\right\}\right]$, point $c$ being an interior point of $I_{0}$. Let a function $F$ be such that

$$
F\left(I_{0}\right)>\omega\left(F,\left[c, x_{0}+\delta\left(x_{0}\right)\right]\right)+\varepsilon \text { for some } \varepsilon>0
$$

$(\omega(F, I)$ denoting the oscillation of $F$ on $I)$. We may suppose also that

$$
\delta(x)<\operatorname{dist}\left(\left(x_{0}-\delta\left(x_{0}\right), x_{0}+\delta\left(x_{0}\right)\right), E \backslash\left\{x_{0}\right\}\right)
$$

if $x \in E \backslash\left\{x_{0}\right\}$. Choose $\pi_{1} \subset B_{\delta}[E \cap[a, c]] \cap B_{\delta}([a, c])$ and $\pi_{2} \subset B_{\delta}[E \cap[c, b]] \cap$ $B_{\delta}([c, b])$ so that

$$
\sum_{(I, x) \in \pi_{1}}|F(I)|>V_{\delta}([a, c])-\frac{\varepsilon}{2} \quad \text { and } \sum_{(I, x) \in \pi_{2}}|F(I)|>V_{\delta}([c, b])-\frac{\varepsilon}{2} .
$$

Let $\left(I^{\prime}, x_{0}\right)$ be an element of $\pi_{2}$ which belongs to $B_{\delta}\left[\left\{x_{0}\right\}\right]$. (We can always suppose that such an element is present in $\pi_{2}$.) In view of $(4) F\left(I_{0}\right)>F\left(I^{\prime}\right)+\varepsilon$. By this and (5) we get

$$
\begin{aligned}
V_{\delta}[a, b] & \geq \sum_{(I, x) \in \pi_{1}}|F(I)|+\sum_{(I, x) \in \pi_{2}}|F(I)|-F\left(I^{\prime}\right)+F\left(I_{0}\right) \\
& >V_{\delta}([a, c])-\frac{\varepsilon}{2}+V_{\delta}([c, b])-\frac{\varepsilon}{2}+\varepsilon=V_{\delta}([a, c])+V_{\delta}([c, b])
\end{aligned}
$$

proving that $V_{\delta}(A)$ is not additive.

A common way to make an additive variation of $V_{\delta}$ is to set $\chi([c, d])=$ $V_{\delta}([a, d])-V_{\delta}([a, c])$ with $a$ fixed. This difference is of course additive but unfortunately we can lose continuity. To observe this we can use an example similar to the previous one. Let $x_{0}$ be a point of $E$ isolated from the left and define $F$ so that $\left|F\left(\left[x_{1}, x_{2}\right]\right)\right|>\varepsilon>0$ for some $x_{1}<x_{0},\left[x_{1}, x_{0}\right] \cap E=\left\{x_{0}\right\}$ and any $x_{2} \geq x_{0}$ where $\left[x_{1}, x_{2}\right] \in B_{\delta}\left[\left\{x_{0}\right\}\right]$. Then for any $c<x_{0} \leq d$ intervals of $B_{\delta}\left[\left\{x_{0}\right\}\right]$ are not represented in any partitions of $B[E \cap[a, c]]$ and are present in partitions of $B[E \cap[a, d]]$. Now having defined $\delta(x)>0$ so that $x+\delta(x)<x_{1}$ for any $x \in E \cap\left[a, x_{0}\right)$ and choosing $\pi \in B_{\delta}[E \cap[a, c]]$ so that

$$
\sum_{(I, x) \in \pi}|F(I)|>V_{\delta}([a, c])-\frac{\varepsilon}{2}
$$


we compute for $c<x_{0} \leq x_{2} \leq d$

$$
V_{\delta}([a, d]) \geq \sum_{(I, x) \in \pi}|F(I)|+\left|F\left(\left[x_{1}, x_{0}\right]\right)\right|>V_{\delta}([a, c])-\frac{\varepsilon}{2}+\varepsilon=V_{\delta}([a, c])+\frac{\varepsilon}{2} .
$$

Thus $\chi([c, d)]>\frac{\varepsilon}{2}$ however small the interval $[c, d]$ might be. (Note that it is at this point where a gap in $[4$, p. 57] occurs. The argument employed there to prove that the function $\chi_{12}$ is continuous does not work for $x$ if it is taken to be an isolated point of the set $M$ as in the example above. A proof in [8] contains a similar gap.)

We are going to overcome these difficulties by introducing for the case of ordinary full covering relation the so called left and right $\delta$-variation variation (see [9]). We put

$$
\begin{aligned}
B_{\delta, l}[E] & =\{([y, x], x): x-\delta(x)<y<x, x \in E\} \\
B_{\delta, r}[E] & =\{([x, z], x): x<z<x+\delta(x), x \in E\} .
\end{aligned}
$$

(These are what in [5, p. 63], are called left-complete and right-complete families.) For a function $F$, for a set $E \subset R$ and an interval $A$ we define

$$
V_{\delta, l}(A)=V\left(B_{\delta, l}, F, E, A\right), V_{\delta, r}(A)=V\left(B_{\delta, r}, F, E, A\right) .
$$

Theorem 1 Let $F$ be a continuous function on an interval $[a, b]$, let $E \subset[a, b]$ and $B_{\delta, l}[E]$ and $B_{\delta, r}[E]$ be fixed and let $V_{\delta, r}([a, b]), V_{\delta, l}([a, b])$ be finite. Then $V_{\delta, r}([a, x])$ and $V_{\delta, l}([x, b])$ are continuous functions at each point $x \in[a, b]$.

Proof. Fix any $x \in[a, b]$ and $\varepsilon>0$. Choose $\eta>0$ so that

$$
|F(I)|<\frac{\varepsilon}{3} \text { if }|I|<\eta \text { and } V_{\delta, r}(I)<\frac{\varepsilon}{3} \text { if } x \in I \text { and }|I|<\eta .
$$

(We are using Proposition 1 for $V_{\delta, r}(A)$.) Take $c, d$ such that

$$
c \leq x \leq d, \quad d-c<\eta
$$

and a partition $\pi \in B_{\delta, r}[E \cap[a, d]] \cap B_{\delta, r}([a, d])$ such that

$$
\sum_{(I, x) \in \pi}|F(I)|>V_{\delta, r}([a, d])-\frac{\varepsilon}{3} .
$$

Let $\pi_{1}=\{(I, x) \in \pi: I \subset[a, c]\}, \pi_{2}=\{(I, x) \in \pi: I \subset[c, d]\}$. By (6) and (7) we have

$$
\sum_{(I, x) \in \pi_{2}}|F(I)| \leq V_{\delta, r}([c, d])<\frac{\varepsilon}{3} .
$$


Let $c \in I_{1}=\left[x_{1}, y_{1}\right]$ where $\left(I_{1}, x_{1}\right) \in \pi$ if such an $I_{1}$ exists. Then once again applying (6) and (7) we get

$$
\left|F\left(I_{1}\right)\right| \leq\left|F\left(\left[x_{1}, c\right]\right)\right|+\left|F\left(\left[c, y_{1}\right]\right)\right|<\left|F\left(\left[x_{1}, c\right]\right)\right|+\frac{\varepsilon}{3} .
$$

Notice that $\pi_{1}^{\prime}=\pi_{1} \cup\left\{\left(\left[x_{1}, c\right], x_{1}\right)\right\} \in B_{\delta, r}[E \cap[a, c]] \cap B_{\delta, r}([a, c])$. We have by (8), (9) and (10)

$$
\begin{aligned}
V_{\delta, r}([a, d]) & <\sum_{(I, x) \in \pi_{1}}|F(I)|+\sum_{(I, x) \in \pi_{2}}|F(I)|+\left|F\left(I_{1}\right)\right|+\frac{\varepsilon}{3} \\
& \leq \sum_{(I, x) \in \pi_{1}}|F(I)|+F\left(\left[x_{1}, c\right]\right)+\frac{\varepsilon}{3}+\sum_{(I, x) \in \pi_{2}}|F(I)|+\frac{\varepsilon}{3} \\
& <\sum_{(I, x) \in \pi_{1}^{\prime}}|F(I)|+\frac{\varepsilon}{3}+\frac{\varepsilon}{3}+\frac{\varepsilon}{3} \leq V_{\delta, r}([a, c])+\varepsilon .
\end{aligned}
$$

Thus $0<V_{\delta, r}([a, d])-V_{\delta, r}([a, c])<\varepsilon$ if $x \in[c, d]$ and $0 \leq d-c<\eta$. This means that $V_{\delta, r}([a, x])$ is continuous as a point-function at the point $x$.

In the same way we can prove the continuity of the point-function $V_{\delta, l}([x, b])$ at any $x \in[a, b]$. This completes the proof of the theorem.

Let $f$ be Henstock integrable (H-integrable) on $[a, b]$ and $F(x)=H-\int_{a}^{x} f$. Then $F^{\prime}(x)=f(x)$ a.e. (see [6] or [5]). Hence the set

$$
P=\left\{x \in[a, b]: F^{\prime}(x) \text { does not exist }\right\}
$$

has measure 0. It is also known (see [6]) that if $g(x)=f(x)$ a.e. on $[a, b]$, then $g$ is also $H$-integrable on $[a, b]$ and $F(x)=H$ - $\int_{a}^{x} g$. Hence we can suppose without lose of generality that

$$
f(x)= \begin{cases}F^{\prime}(x) & \text { if } x \notin P \\ 0 & \text { if } x \in P\end{cases}
$$

Lemma 1 If the set $P$ is defined by (11), then for any $\varepsilon>0$ there exists $\delta(x)>0$ defined on $P$ such that

$$
V_{\delta, r}([a, b])=V\left(B_{\delta, r}, F, P,[a, b]\right)<\frac{\varepsilon}{4}
$$

and

$$
V_{\delta, l}([a, b])=V\left(B_{\delta, l}, F, P,[a, b]\right)<\frac{\varepsilon}{4} .
$$


Proof. This property is known for full covering relation $B_{\delta}$ as the Strong Lusin condition (see [7] or [3]). Hence Lemma 1 follows from the fact that both $B_{\delta, r}$ and $B_{\delta, l}$ are subsets of $B_{\delta}$.

We are now prepared to describe a construction of continuous major and minor functions of a Henstock integrable function.

Theorem 2 Let a function $f$ defined by (12) be $H$-integrable on $[a, b]$ with $F(x)=H-\int_{a}^{x} f$ and let $P$ be defined by (11). Let $\delta(x)>0$ be defined by Lemma 1 for preassigned $\varepsilon>0$. Then functions

$$
\begin{aligned}
M(x) & =F(x)+V_{\delta, r}([a, x])+V_{\delta, l}([a, b])-V_{\delta, l}([x, b]) \\
m(x) & =F(x)-V_{\delta, r}([a, x])-V_{\delta, l}([a, b])+V_{\delta, l}([x, b])
\end{aligned}
$$

are continuous major and minor functions respectively and

$$
M(b)-m(b)<\varepsilon .
$$

Proof. Condition (13) follows from Lemma 1. Continuity of $M$ and $m$ follows from Theorem 1. The superadditivity of $V_{\delta, r}(A)$ and $V_{\delta, l}(A)$ implies

$$
V_{\delta, r}([a, d])-V_{\delta, r}([a, c]) \geq V_{\delta, r}([c, d]) \geq|F(d)-F(c)|
$$

if $c \in P$ and $0<d-c<\delta(c)$, and

$$
V_{\delta, l}([c, b])-V_{\delta, l}([d, b]) \geq V_{\delta, l}([c, d]) \geq|F(d)-F(c)|
$$

if $d \in P$ and $0<d-c<\delta(d)$. So if $x \in P$ and $0<h<\delta(x)$, then

$$
\begin{aligned}
M(x+h)-M(x) \geq & F(x+h)-F(x)+|F(x+h)-F(x)| \\
& -V_{\delta, l}([x+h, b])+V_{\delta, l}([x, b]) \geq 0
\end{aligned}
$$

and similarly for the same $h M(x)-M(x-h) \geq 0$. Hence $\underline{D} M(x) \geq 0=$ $f(x)$ if $x \in P$. At the same time obviously $\underline{D} M(x) \geq F^{\prime}(x)=f(x)$ if $x \in$ $[a, b] \backslash P$. Therefore $M$ is a continuous major function of $f$. In the same way we can check that $m$ is a continuous minor function of $f$.

\section{References}

[1] P. S. Bullen et al (ed), New Integrals, Springer-Verlag, Lecture Notes, 1419 (1990).

[2] Z. T. Gong, On a problem of Skvortsov involving the Perron Integral, Real Analysis Exchange, 17 no. 2 (1991-92), 748-750. 
[3] R. Gordon, A descriptive characterization of the generalized Riemann Integral, Real Analysis Exchange, 15 no. 1 (1989-90), 397-400.

[4] R. Henstock, Majorants in variational integration, Canadian Journal of Math., 18 (1966), 49-74.

[5] R. Henstock, Theory of integration, Butterworth, London, 1963.

[6] P. Y. Lee, Lanzhou Lecture on Henstock integration, World Scientific Press, 1989.

[7] P. Y. Lee and R. Výborný, Kurzweil-Henstock integration and the strong Lusin condition, Bullettino U.M.I., 7, 7-B (1993), 761-773.

[8] G. Liu, P. Y. Lee and P. S. Bullen, A note on major and minor function for the Perron integral, Real Analysis Exchange, 20 no. 1, (1994-95), 336-339.

[9] S. Lu, On the construction of major and minor functions, Journal of Math. Study, 27 no. 1 (1994), 121-126.

[10] K. M. Ostaszewski, Henstock integration in the plane, AMS Memoirs, 353 (1986).

[11] S. Saks, Theory of the integral, Dover, New York, 1964.

[12] V. A. Skvortsov and B. S. Thomson, Symmetric integrals do not have the Marcinkiewicz property, Real Analysis Exchange, to appear.

[13] B. S. Thomson, Derivates of interval functions, AMS Memoirs, 452 (1991). 